\title{
In Silico Functional Assessment of Sequence Variations: Predicting Phenotypic Functions of Novel Variations
}

\author{
Hong-Hee Won ${ }^{1,2}$ and Jong-Won $\mathrm{Kim}^{3 *}$ \\ ${ }^{1}$ Samsung Biomedical Research Institute, Samsung Me- \\ dical Center, Seoul 135-710, Korea, ${ }^{2}$ Department of Bio \\ and Brain Engineering, Korea Advanced Institute of Sci- \\ ence and Technology, Daejeon 305-701, Korea, ${ }^{3}$ De- \\ partment of Laboratory Medicine and Genetics, Sung- \\ kyunkwan University School of Medicine, Samsung \\ Medical Center, Seoul 135-710, Korea
}

\begin{abstract}
A multitude of protein-coding sequence variations (CVs) in the human genome have been revealed as a result of major initiatives, including the Human Variome Project, the 1000 Genomes Project, and the International Cancer Genome Consortium. This naturally has led to debate over how to accurately assess the functional consequences of $\mathrm{CVs}$, because predicting the functional effects of CVs and their relevance to disease phenotypes is becoming increasingly important. This article surveys and compares variation databases and in silico prediction programs that assess the effects of CVs on protein function. We also introduce a combinatorial approach that uses machine learning algorithms to improve prediction performance.
\end{abstract}

Keywords: sequence variation, amino acid substitution, nonsynonymous single nucleotide polymorphism, missense mutation, prediction, protein function

\section{Introduction}

Single amino acid substitutions in protein-coding sequences are common in the human genome. These protein-coding sequence variations (CVs) are important diagnostic markers and therapeutic targets in genetic disease studies. Although most CVs are functionally neutral, some CVs affect phenotype, including nonsynonymous single nucleotide polymorphisms (nsSNPs) that contribute to normal phenotypic differences in hair color, skin color (Sulem et al., 2007; Han et al., 2008), and disease susceptibility (WTCCC, 2007; Amos et al., 2008; Harley et al., 2008; Tenesa et al., 2008). Other

*Corresponding author: E-mail kimjw@skku.edu

Tel +82-2-3410-2705, Fax +82-2-3410-2719

Accepted 25 November 2008
CVs result in deleterious missense mutations that cause highly penetrant Mendelian-inherited diseases (Kim et al., 2007). These deleterious mutations have been of great interest in biomedical research and clinical practice for decades and account for approximately half of the genetic variations that are known to cause disease.

Using recent advancements in sequencing technologies, several studies have reported a number of sequence variations in certain cancers (Sjoblom et al., 2006; Greenman et al., 2007; Campbell et al., 2008; Jones et al., 2008), in which mutational patterns have differed greatly between patients with the same disease. Furthermore, major initiatives, such as the Human Variome Project, the 1000 Genomes Project, and the International Cancer Genome Consortium, will generate a vast amount of variation data. Consequently, it is important to assess variations in conjunction with protein function and disease phenotype. Several databases, such as the Online Mendelian Inheritance in Man (OMIM) and the Human Gene Mutation Database (HGMD), have documented CVs that correspond to Mendelian-inherited human diseases. In addition, many computational programs have been created to predict the functional effects of unknown CVs ( $\mathrm{Ng}$ et al., 2006; Care et al., 2007). Database searches and bioinformatic predictions can be useful in prioritizing novel CVs for further analysis.

In this review, we summarize the databases that are most helpful in interpreting the functional effects of CVs. We perform an extensive survey of existing in silico prediction methods and compare their performance. Finally, we introduce a combination method as a promising approach to improve prediction performance.

\section{Polymorphism and Mutation Databases}

Several databases that are helpful in assessing the functional effects of CVs or their relevance to disease phenotype are listed in Table 1. Each of two broad-category mutation databases, general mutation databases (GMDBs) and locus-specific mutation databases (LSDBs), has unique strengths and weaknesses (Porter et al., 2000). Because polymorphism and mutation databases have been developed for different uses, they complement each other. 
Table 1. Polymorphism and mutation databases

\begin{tabular}{|c|c|c|c|c|}
\hline Database & Recent release date ${ }^{*}$ & Data type & Features & Website \\
\hline $\begin{array}{l}\text { OMIM } \\
\text { (Hamosh et al., } \\
\text { 2005) }\end{array}$ & Updated daily & Deleterious mutations & $\begin{array}{l}\text { Full-text descriptions of published } \\
\text { disease-causing variations }\end{array}$ & $\begin{array}{l}\text { http://www.ncbi.nlm.nih. } \\
\text { gov/omim }\end{array}$ \\
\hline $\begin{array}{l}\text { HGMD } \\
\text { (Stenson et al., 2008) }\end{array}$ & Sept 2008 & Deleterious mutations & $\begin{array}{l}\text { Comprehensive collection of published } \\
\text { disease-causing variations }\end{array}$ & http://www.hgmd.cf.ac.uk \\
\hline LSDB in HGVS & Nov 2008 & Deleterious mutations & $\begin{array}{l}\text { Specialized collection of a particular } \\
\text { gene or locus }\end{array}$ & $\begin{array}{l}\text { http://www.hgvs.org/dblist/ } \\
\text { glsdb.html }\end{array}$ \\
\hline $\begin{array}{l}\text { Swiss-Prot } \\
\text { (Yip et al., 2004) }\end{array}$ & Nov 2008 & $\begin{array}{l}\text { Deleterious mutations } \\
\text { and neutral } \\
\text { polymorphisms }\end{array}$ & $\begin{array}{l}\text { Well-summarized list of variations and } \\
\text { corresponding proteins }\end{array}$ & $\begin{array}{l}\text { http://www.expasy.org/ } \\
\text { cgi-bin/lists?humsavar.txt }\end{array}$ \\
\hline $\begin{array}{l}\mathrm{dbSNP} \\
\text { (Sherry et al., 2001) }\end{array}$ & Apr 2008 & $\begin{array}{l}\text { Neutral and (few) } \\
\text { deleterious SNPs }\end{array}$ & $\begin{array}{l}\text { Broad collections of SNPs regardless } \\
\text { of clinical associations (clinically } \\
\text { associated SNPs linked to source } \\
\text { sites) }\end{array}$ & $\begin{array}{l}\text { http://www.ncbi.nlm, nih. } \\
\text { gov/projects/SNP }\end{array}$ \\
\hline $\begin{array}{l}\mathrm{dbGaP} \\
\text { (Mailman et al., } \\
\text { 2007) }\end{array}$ & Nov 2008 & $\begin{array}{l}\text { Deleterious or pheno- } \\
\text { type-affecting SNPs }\end{array}$ & $\begin{array}{l}\text { Collections of SNPs affecting clinical } \\
\text { phenotypes or nonclinical traits }\end{array}$ & $\begin{array}{l}\text { http://www.ncbi.nlm.nih. } \\
\text { gov/gap }\end{array}$ \\
\hline $\begin{array}{l}\text { HapMap } \\
\text { (Frazer et al., 2007) }\end{array}$ & Oct 2008 & $\begin{array}{l}\text { Neutral SNPs and (very } \\
\text { few) deleterious SNPs }\end{array}$ & $\begin{array}{l}\text { Collections of SNPs of } 270 \text { individuals } \\
\text { randomly selected from African, } \\
\text { Asian, and European populations }\end{array}$ & http://www.hapmap.org \\
\hline
\end{tabular}

*Accessed Nov 2008. Because LSDBs are individually updated, the presented release date is for HGVS.

\section{OMIM}

OMIM is among the most representative and well-documented GMDBs, and it contains a full-text overview of human genes and genetic disorders. The contents of OMIM are considered comprehensive, authoritative, and timely, because they are written at Johns Hopkins University School of Medicine and edited daily with input from scientists and physicians from around the world (Hamosh et al., 2005). Many CVs that are registered in OMIM may have deleterious effects on protein function and cause Mendelian-inherited diseases. OMIM also includes some disease susceptibility variations that are found in association analyses. OMIM is therefore a valuable resource to study the characteristics of variations that severely affect a certain phenotype.

\section{HGMD}

HGMD is a comprehensive and publicly available GMDB of gene lesions that underlie human inherited diseases (Krawczak et al., 2000; Stenson et al., 2003; Stenson et al., 2008). Two different versions of the database exist: an up-to-date commercial version and an older public version-both of them provide comprehensive mutation information. The total number of public entries that are available to users from academic institutions or nonprofit organizations is 61,447 , comprising 35,168 missense or nonsense mutations, 10,035 small deletions, and 5805 splicing mutations. Because data are collected by a combination of manual and computerized searches, the contents are considered highly reliable. GMDBs, such as OMIM and HGMD, have several limitations, however, most of which are attributable to less-specialized knowledge of particular genetic loci (Porter et al., 2000).

\section{LSDB and Swiss-Prot}

LSDBs are usually maintained by experts on a particular gene or locus, provide a greater depth of information about each variation, and often present unpublished data that are submitted directly by researchers in related fields. In contrast, LSDBs can often become stagnant or disappear because they are likely to depend on limited funding resources and part-time scientists (Porter et al., 2000). Hundreds of LSDBs for 718 genes are currently listed on the website of the Human Genome Variation Society (HGVS). The Swiss-Prot database (release version 56.4), with 46,799 CVs for 9085 proteins, complements LSDBs. These CVs are particularly useful for developing prediction algorithms of functionality (Care et al., 2007), because Swiss-Prot provides a well-summarized list of CVs with corresponding protein identifications, sequence positions, amino acid changes, and disease associations (disease $v s$. polymorphism). 


\section{dbSNP}

NCBl's single nucleotide polymorphisms database (dbSNP, build 129) contains 14.7 million human reference SNPs. Their broad collection of simple genetic polymorphisms includes SNPs, small-scale multibase deletions or insertions, retrotransposable element insertions, and microsatellite repeat variations (Sherry et al., 2001). The database provides the frequency of the polymorphism by population or individual, allowing for estimates of prevalence in a specific population. The database archives variations regardless of their clinical associations and contains some clinically associated SNPs that are linked to OMIM, LSDB, or the clinical laboratory. It should be noted that SNPs that lack clinical associations and have not been functionally validated may still be relevant.

\section{$\mathrm{dbGaP}$}

The database of genotype and phenotype (dbGaP) archives the results of studies that investigate the interaction between genotype and phenotype (Mailman et al., 2007). The database includes SNPs that affect both clinical and nonclinical phenotypes that are found in genome-wide association studies, medical sequencing, and molecular diagnostic assays. The results are categorized by study and by disease. More than 30 studies are listed, each comprising thousands of case-control sets or parent-offspring trios. Authorized users can download individual-level data for their own research.

\section{HapMap}

The haplotype map (HapMap) (release \#24) contains the genotypes and frequencies of over 3.8 million SNPs. The SNPs were obtained by analyzing DNA samples from 270 individuals, comprising 30 trios of two parents and an adult child of African ancestry, 30 trios of European ancestry, and 90 unrelated individuals of Asian ancestry (Frazer et al., 2007). Because the individuals were randomly selected, one would expect that very few variations in HapMap are functionally deleterious; therefore, HapMap data could be used as a reference set of neutral CVs.

In conclusion, the OMIM, HGMD, and LSDB databases catalog known deleterious mutations that result in severe disease, while the Swiss-Prot and dbGaP databases record those that have modest effects on the resulting protein. The Swiss-Prot, dbSNP, and HapMap databases provide fundamental information on neutral polymorphisms.

\section{Prediction Programs for Functional As- sessment of Sequence Variations}

Because it was shown that protein structure and sequence-based attributes could provide information to distinguish deleterious mutations from neutral single-base changes (Sunyaev et al., 2000; Chasman et al., 2001), many prediction programs have been developed and implemented on a web server to provide in silico prediction of CV functionality ( $\mathrm{Ng}$ et al., 2006; Care et al., 2007). These programs employ a variety of rulebased models and machine learning algorithms, using information on protein structure, sequence, physicochemical properties, phylogenetics, and evolutionary features. The widely used programs are listed below and summarized in Table 2.

\section{SIFT and PolyPhen}

The program Sorting Intolerant From Tolerant (SIFT) uses sequence homology to calculate a scaled probability for the substitution that is observed ( $\mathrm{Ng}$ et al., 2001; Ng et al., 2002; Ng et al., 2003). Substitutions that have a low scaled probability are predicted to affect protein function. The Swiss-Prot/TrEMBL databases and PSI-BLAST were used for sequence alignment. As the first program that was implemented on a web server, SIFT is one of the most frequently used, along with Polymorphism Phenotyping (PolyPhen) (Sunyaev et al., 2001; Ramensky et al., 2002). PolyPhen uses empirically derived rules to predict the effect of $\mathrm{CVs}$ on protein function. The rules are based on known protein structures, sequence conservation between homologous proteins, and sequence-based characterization of the substitution site (e.g., binds lipid, metal). These two programs are among the earliest developed programs and have been recently updated.

\section{MSRV}

A method that was published by Jiang et al. adopts Multiple Selection Rule Voting (MSRV), which includes three physicochemical properties (molecular weight, pl value, and hydrophobicity scale) of amino acids, three relative frequencies for the presence of amino acids in secondary structures (helices, strands, and turns), and two evolutionary conservation scores (Jiang et al., 2007). These authors compared the areas under the receiver operating curves (AUCs) of MSRV, SIFT, and PolyPhen and showed that MSRV employs optimal feature sets, outperforming SIFT and PolyPhen in prioritizing disease mutations that are responsible for monogenic and polygenic diseases. 
Table 2. Programs for predicting functional effects of coding sequence variations

\begin{tabular}{|c|c|c|c|c|c|}
\hline Method & $\begin{array}{c}\text { Recent } \\
\text { release date* }\end{array}$ & Algorithm & Performance $^{\dagger}$ & $\begin{array}{l}\text { Source } \\
\text { code }\end{array}$ & Website \\
\hline $\begin{array}{l}\text { SIFT } \\
\text { (Ng et al., 2003) }\end{array}$ & March 2008 & $\begin{array}{l}\text { Calculates a scaled probability for } \\
\text { the substitution using sequence } \\
\text { homology }\end{array}$ & $\begin{array}{l}\text { FN error: } 31 \% \\
\text { FP error: } 20 \%\end{array}$ & Available & http://blocks.fhcrc.org/sift \\
\hline $\begin{array}{l}\text { PolyPhen } \\
\text { (Ramensky } \\
\text { et al., 2002) }\end{array}$ & March 2008 & $\begin{array}{l}\text { Empirical rules based on } \\
\text { characterization of the substitution } \\
\text { site, conservation between } \\
\text { homologous proteins, and protein } \\
\text { structures }\end{array}$ & $\begin{array}{l}\text { FN error: } 31 \% \\
\text { FP error: } 9 \%\end{array}$ & On request & http://coot.embl.de/PolyPhen \\
\hline $\begin{array}{l}\text { MSRV } \\
\text { (Jiang et al., } \\
2007 \text { ) }\end{array}$ & Aug 2007 & $\begin{array}{l}\text { Multiple Selection Rule Voting (MSRV) } \\
\text { using physicochemical properties, } \\
\text { relative frequencies in secondary } \\
\text { structures, and evolutionary } \\
\text { conservation }\end{array}$ & $\begin{array}{l}\text { AUC: } 82 \sim 87 \% \\
\text { SIFT AUC: } 75 \% \\
\text { PolyPhen AUC: } \\
\text { 70 } 75 \%\end{array}$ & $\begin{array}{l}\text { Not } \\
\text { specified }\end{array}$ & http://msms.usc.edu/msrv \\
\hline $\begin{array}{l}\text { PANTHER } \\
\text { (Thomas et al., } \\
\text { 2003) }\end{array}$ & Aug 2007 & $\begin{array}{l}\text { Calculates the functional likelihood } \\
\text { using a hidden Markov model with } \\
\text { a protein family library }\end{array}$ & $\begin{array}{l}\text { FN error: } 59 \% \\
\text { FP error: } N / A\end{array}$ & Available & $\begin{array}{l}\text { http://www.pantherdb.org/tools/ } \\
\text { csnpScoreForm.jsp }\end{array}$ \\
\hline $\begin{array}{l}\text { SNAP } \\
\text { (Bromberg et } \\
\text { al., 2007) }\end{array}$ & Sept 2008 & $\begin{array}{l}\text { Combines sequence analysis tools } \\
\text { and uses protein annotation, solvent } \\
\text { accessibility, secondary structure, } \\
\text { flexibility, SIFT results, and } \\
\text { conservation }\end{array}$ & $\begin{array}{l}\text { FN error: } 20 \% \\
\text { FP error: } 24 \%\end{array}$ & On request & http://rostlab.org/services/SNAP \\
\hline $\begin{array}{l}\text { PMUT } \\
\text { (Ferrer-Costa } \\
\text { et al., 2005) }\end{array}$ & May 2005 & $\begin{array}{l}\text { Uses two neural networks trained with } \\
\text { human mutation data using structural } \\
\text { and evolutionary information }\end{array}$ & $\begin{array}{l}\text { FN error: } 12 \sim 21 \% \\
\text { FP error: } 10 \sim 17 \%\end{array}$ & $\begin{array}{l}\text { Not } \\
\text { specified }\end{array}$ & $\begin{array}{l}\text { http://mmb2.pcb.ub.es:8080/ } \\
\text { PMut }\end{array}$ \\
\hline $\begin{array}{l}\text { nsSNPAnalyzer } \\
\text { (Bao et al., } \\
\text { 2005) }\end{array}$ & Feb 2005 & $\begin{array}{l}\text { Random forest trained with structural } \\
\text { information, sequence conservation, } \\
\text { and SIFT prediction using Swiss-Prot } \\
\text { data }\end{array}$ & $\begin{array}{l}\text { FN error: } 21 \% \\
\text { FP error: } 38 \%\end{array}$ & Available & http://snpanalyzer.utmem.edu \\
\hline
\end{tabular}

*Accessed Nov 2008. If the release date was not available on the website, the publication date was presented.

${ }^{\dagger}$ Performance was summarized based on the literature in which the method was introduced. False negative (FN) error rate is the percentage of the deleterious variations predicted to be neutral. False positive (FP) error rate is the percentage of the neutral variations predicted to be deleterious. Area under the receiver operating characteristic curve (AUC) was calculated in the Jiang et al. study (Jiang et al., 2007).

\section{PANTHER}

The program PANTHER can predict the effect of CVs on protein function by relating sequence to function (Thomas et al., 2003; Thomas et al., 2004). The program uses a hidden Markov model and a library of protein families to score the functional likelihood of different amino acid substitutions. The phenotypic effect is determined by the position-specific evolutionary conservation (PSEC) scores that are obtained from the model. Because the source codes for the PANTHER predictor and the PANTHER library are available online, this method is useful for analyzing a large number of CVs.

\section{SNAP and PMUT}

Screening for Non-Acceptable Polymorphisms (SNAP) predicts non-neutral substitutions by using annotations from the protein mutant database (Kawabata et al., 1999) and by combining many sequence analysis tools in neural networks (NNs) (Bromberg et al., 2007; Bromberg et al., 2008a; Bromberg et al., 2008b). It also uses solvent accessibility, secondary structure, flexibility, SIFT results, and conservation information. SNAP gives a reliability index of the prediction, ranging from 0 (low) to 9 (high reliability). PMUT is similar to SNAP with regard to its methods and output. PMUT is also based on the use of two NNs that are trained with human mutation data (Ferrer-Costa et al., 2002; Ferrer-Costa et al., 2004; Ferrer-Costa et al., 2005). It displays a pathogenicity index that ranges from 0 to 1 (>0.5 signals pathological mutations), a confidence index that ranges from 0 (low) to 9 (high confidence), and the mutation site on the protein structure to trace its pathogenicity. 


\section{nsSNPAnalyzer}

The program nsSNPAnalyzer uses a machine learning algorithm called random forest to combine heterogeneous sources of predictors (Bao et al., 2005; Bao et al., 2005). Random forest was trained with various features, such as structural information, sequence conservation, and SIFT prediction using a dataset from Swiss-Prot. The source codes of nsSNPAnalyzer are also available on the website.

As reviewed in this section, many programs use common sources and methods while exploiting different algorithms, features, and databases. Combined use of these in silico programs is encouraged to mitigate their limitations in prediction performance. Source codes are necessary to analyze large-scale data with several programs; code availability is summarized in Table 2.

\section{Combination Approach to Predicting Func- tion}

Given that predictions of the functional consequences of amino acid substitutions can be more accurate by combining different in silico methods ( $\mathrm{Ng}$ et al., 2006), a combination approach has been proposed to improve prediction accuracy (Won et al., 2008). To assess the

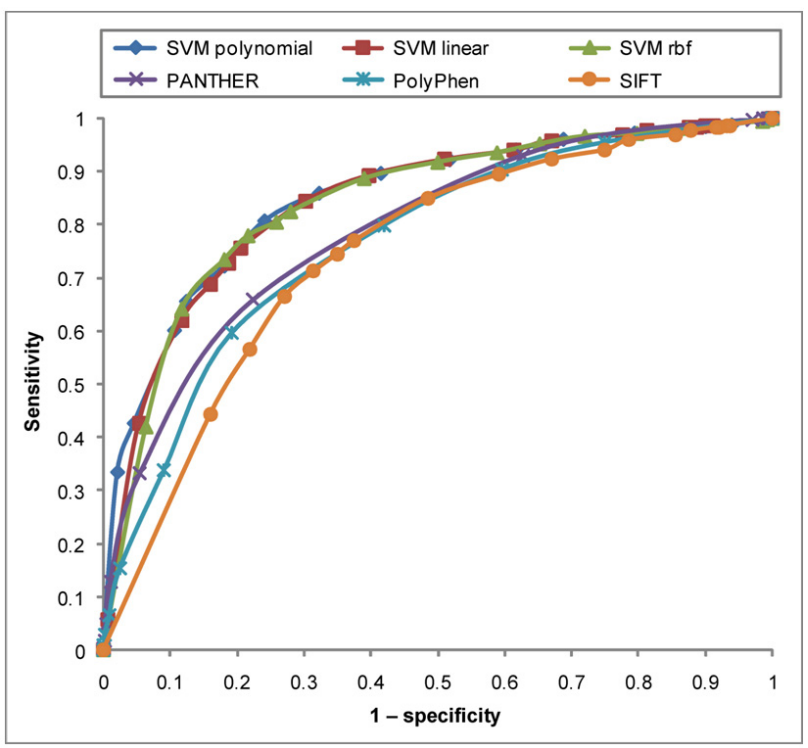

Fig. 1. Receiver operating characteristic (ROC) curves of individual predictors and their combinations. The ROC curves of SVM combinations tend toward the upper left corner of the plot more than the three individual prediction programs, indicating superior performance. This shows that the appropriate combination can noticeably improve prediction accuracy. Figure from Won et al., 2008. effectiveness of this approach, the prediction performance of individual programs must be evaluated. A support vector machine (SVM) was used to combine three representative in silico prediction programs (SIFT, PolyPhen, and PANTHER) to predict the phenotypic effects of CVs.

Assuming that the HapMap dataset comprises mainly nonpathogenic variations (negative samples) while the HGMD dataset comprises pathogenic variations (positive samples), we compared the prediction performances of SVM combinations and individual predictors, including SIFT, PolyPhen, and PANTHER (see Won et al., 2008 for details). The three different kernel functions-a linear kernel, a polynomial kernel, and a radial basis function kernel-were used to train SVMs. The experimental results show that the SVM combinations outperform the individual prediction programs (Fig. 1). In particular, $\mathrm{SVM}_{\text {polynomial }}$ has a slightly superior predictive value than the other two SVM combinations. PANTHER outperforms PolyPhen and SIFT in terms of sensitivity over all specificity regions. The superior performance of $S \mathrm{M}_{\text {polynomial }}$ indicates that the appropriate combination can effectively improve prediction accuracy.

\section{Conclusion}

Interpreting the functionality of newly found variations in gene coding regions is of much importance to both biomedical research and clinical practice. The first step to understanding these variations is to examine them using valuable resources, such as variation databases and functional prediction programs. Furthermore, automated prediction methods are essential for analyzing CVs on a genome-wide scale. This review summarizes representative examples of useful resources and emphasizes the ongoing need for improvement in the performance of individual prediction methods. We suggest that comprehensive analyses that use a combination of complementary databases and in silico programs are necessary to overcome the relative weakness of each program. In the case of SVM combinations, we showed that prediction can be improved effectively if the results of the individual programs are appropriately combined.

\section{Acknowledgments}

This study was supported by the Korean HapMap Project, funded by the Korean Ministry of Education, Science and Technology.

\section{References}

Amos, C.I., Wu, X., Broderick, P., et al. (2008). Genome- 
wide association scan of tag SNPs identifies a susceptibility locus for lung cancer at 15q25.1. Nat. Genet. 40, 616-622

Bao, L., and Cui, Y. (2005). Prediction of the phenotypic effects of non-synonymous single nucleotide polymorphisms using structural and evolutionary information. Bioinformatics 21, 2185-2190.

Bao, L., Zhou, M., and Cui, Y. (2005). nsSNPAnalyzer: identifying disease-associated nonsynonymous single nucleotide polymorphisms. Nucleic Acids Res. 33(Web Server issue), W480-482.

Bromberg, Y., and Rost, B. (2007). SNAP: predict effect of non-synonymous polymorphisms on function. Nucleic Acids Res. 35, 3823-3835.

Bromberg, Y., and Rost, B. (2008a). Comprehensive in silico mutagenesis highlights functionally important residues in proteins. Bioinformatics 24, i207-212.

Bromberg, Y., Yachdav, G., and Rost, B. (2008b). SNAP predicts effect of mutations on protein function. Bioinformatics 24, 2397-2398.

Campbell, P.J., Pleasance, E.D., Stephens, P.J., et al. (2008). Subclonal phylogenetic structures in cancer revealed by ultra-deep sequencing. Proc. Natl. Acad. Sci. U. S. A. 105, 13081-13086

Care, M.A., Needham, C.J., Bulpitt, A.J., and Westhead, D.R. (2007). Deleterious SNP prediction: be mindful of your training data! Bioinformatics 23, 664-672.

Chasman, D., and Adams, R.M. (2001). Predicting the functional consequences of non-synonymous single nucleotide polymorphisms: structure-based assessment of amino acid variation. J. Mol. Biol. 307, 683-706.

Ferrer-Costa, C., Gelpi, J.L., Zamakola, L., et al. (2005). PMUT: a web-based tool for the annotation of pathological mutations on proteins. Bioinformatics 21, 3176-3178

Ferrer-Costa, C., Orozco, M., and de la Cruz, X. (2002). Characterization of disease-associated single amino acid polymorphisms in terms of sequence and structure properties. J. Mol. Biol. 315, 771-786.

Ferrer-Costa, C., Orozco, M., and de la Cruz, X. (2004). Sequence-based prediction of pathological mutations. Proteins 57, 811-819.

Frazer, K.A., Ballinger, D.G., Cox, D.R., et al. (2007). A second generation human haplotype map of over 3.1 million SNPs. Nature 449, 851-861.

Greenman, C., Stephens, P., Smith, R., et al. (2007). Patterns of somatic mutation in human cancer genomes. Nature 446, 153-158.

Hamosh, A., Scott, A.F., Amberger, J.S., et al. (2005). Online Mendelian Inheritance in Man (OMIM), a knowledgebase of human genes and genetic disorders. Nucleic Acids Res. 33(Database issue), D514-517.

Han, J., Kraft, P., Nan, H., et al. (2008). A genome-wide association study identifies novel alleles associated with hair color and skin pigmentation. PLOS Genet. 4, e1000074

Harley, J.B., Alarcon-Riquelme, M.E., Criswell, L.A., et al. (2008). Genome-wide association scan in women with systemic lupus erythematosus identifies susceptibility var- iants in ITGAM, PXK, KIAA1542 and other loci. Nat. Genet. 40, 204-210.

Jiang, R., Yang, H., Zhou, L., et al. (2007). Sequence-based prioritization of nonsynonymous single-nucleotide polymorphisms for the study of disease mutations. Am. J. Hum. Genet. 81, 346-360.

Jones, S., Zhang, X., Parsons, D.W., et al. (2008). Core signaling pathways in human pancreatic cancers revealed by global genomic analyses. Science 321, 1801-1806.

Kawabata, T., Ota, M., and Nishikawa, K. (1999). The protein mutant database. Nucleic Acids Res, 27, 355-357.

Kim, H.J., Sohn, K.M., Shy, M.E., et al. (2007). Mutations in PRPS1, which encodes the phosphoribosyl pyrophosphate synthetase enzyme critical for nucleotide biosynthesis, cause hereditary peripheral neuropathy with hearing loss and optic neuropathy (cmtx5). Am. J. Hum. Genet. 81, 552-558

Krawczak, M., Ball, E.V., Fenton, I., et al. (2000). Human gene mutation database-a biomedical information and research resource. Hum. Mutat. 15, 45-51.

Mailman, M.D., Feolo, M., Jin, Y., et al. (2007). The NCBI $\mathrm{dbGaP}$ database of genotypes and phenotypes. Nat. Genet. 39, 1181-1186.

Ng, P.C., and Henikoff, S. (2001). Predicting deleterious amino acid substitutions. Genome Res. 11, 863-874.

$\mathrm{Ng}$, P.C., and Henikoff, S. (2002). Accounting for human polymorphisms predicted to affect protein function. Genome Res 12, 436-446.

Ng, P.C., and Henikoff, S. (2003). SIFT: Predicting amino acid changes that affect protein function. Nucleic Acids Res. 31, 3812-3814.

Ng, P.C., and Henikoff, S. (2006). Predicting the effects of amino acid substitutions on protein function. Annu. Rev. Genomics Hum. Genet. 7, 61-80.

Porter, C.J., Talbot, C.C., and Cuticchia, A.J. (2000). Central mutation databases--a review. Hum. Mutat. 15, 36-44.

Ramensky, V., Bork, P., and Sunyaev, S. (2002). Human non-synonymous SNPs: server and survey. Nucleic Acids Res. 30, 3894-3900.

Sherry, S.T., Ward, M.H., Kholodov, M., et al. (2001). dbSNP: the NCBI database of genetic variation. Nucleic Acids Res. 29, 308-311.

Sjoblom, T., Jones, S., Wood, L.D., et al. (2006). The consensus coding sequences of human breast and colorectal cancers. Science 314, 268-274.

Stenson, P.D., Ball, E., Howells, K., et al. (2008). Human Gene Mutation Database: towards a comprehensive central mutation database. J. Med. Genet. 45, 124-126.

Stenson, P.D., Ball, E.V., Mort, M., et al. (2003). Human Gene Mutation Database (HGMD): 2003 update. Hum. Mutat. 21, 577-581.

Sulem, P., Gudbjartsson, D.F., Stacey, S.N., et al. (2007). Genetic determinants of hair, eye and skin pigmentation in Europeans. Nat. Genet. 39, 1443-1452.

Sunyaev, S., Ramensky, V., and Bork, P. (2000). Towards a structural basis of human non-synonymous single nucleotide polymorphisms. Trends Genet. 16, 198-200.

Sunyaev, S., Ramensky, V., Koch, I., et al. (2001). 
172 Genomics \& Informatics Vol. 6(4) 166-172, December 2008

Prediction of deleterious human alleles. Hum. Mol. Genet. 10, 591-597.

Tenesa, A., Farrington, S.M., Prendergast, J.G., et al. (2008). Genome-wide association scan identifies a colorectal cancer susceptibility locus on 11q23 and replicates risk loci at 8q24 and 18q21. Nat. Genet. 40, 631-637.

Thomas, P.D., Campbell, M.J., Kejariwal, A., et al. (2003). PANTHER: a library of protein families and subfamilies indexed by function. Genome Res. 13, 2129-2141.

Thomas, P.D., and Kejariwal, A. (2004). Coding single-nucleotide polymorphisms associated with complex vs. Mendelian disease: evolutionary evidence for differ- ences in molecular effects. Proc. Natl, Acad. Sci. U. S. A. 101, 15398-15403.

Won, H.H., Kim, H.J., Lee, K.A., and Kim, J.W. (2008). Cataloging coding sequence variations in human genome databases. PLOS ONE 3, e3575.

WTCCC. (2007). Genome-wide association study of 14,000 cases of seven common diseases and 3,000 shared controls. Nature 447, 661-678.

Yip, Y.L., Scheib, H., Diemand, A.V., et al. (2004). The Swiss-Prot variant page and the ModSNP database: a resource for sequence and structure information on human protein variants. Hum. Mutat. 23, 464-470. 